Originales

\title{
Registro de cáncer de cabeza y cuello: estudio prospectivo de incidencia a dos años
}

J. Granell Navarro*, A. Puig Rullán**

\section{Resumen}

- Propósito: determinar la incidencia del Cáncer de Cabeza y Cuello (CCC) en el área de salud de Puertollano y evaluar la calidad del Registro de CCC.

- Material y métodos: estudio observacional, longitudinal y prospectivo a dos años, basado en el Registro de CCC sobre una población de referencia de 80.000 habitantes.

- Resultados: se diagnosticaron 48 casos (tasa bruta de incidencia anual: 30 por 100.000 habitantes). Todos los tumores de la vía aerodigestiva superior, excepto uno, fueron carcinomas epidermoides y se localizaron 11 en cavidad oral/orofaringe y 21 en laringe/hipofaringe; encontramos 7 cánceres de tiroides, dos tumores malignos de glándula parótida y 7 linfomas.

- Conclusiones: destaca la elevada incidencia del cáncer de laringe (tasa ajustada en varones: 13,3). Los indicadores de calidad de registro fueron excelentes.

Palabras clave:

Registro de cáncer. Incidencia del cáncer de cabeza y cuello.

Oncología, 2004; 27 (1):21-27

* Servicio de Otorrinolaringología

** Servicio de Anatomía Patológica

Hospital Santa Bárbara. Puertollano (Ciudad Real) 


\section{Summary}

- Purpose: To assess the incidence of head and neck cancer (HNC) in the Puertollano area, and to evaluate the quality of the HNC Registry.

- Material and methods: An observational, longitudinal and prospective study of a two-year based HNC Registry for a reference population of 80,000 inhabitants was made.

- Results: Forty-eight cases of HNC were diagnosed, corresponding to an annual incidence rate of $30 / 100,000$. Most of the aerial and digestive tumors were epidermoid carcinomas, 11 were located in the oral cavity and oropharynx, and 21 in the larynx and hypopharynx, 7 were thyroid gland cancers, 2 parotid gland malignant tumors, and 7 lymphomas.

- Conclusions: It is remarkable the high incidence of laryngeal cancer (adjusted male rate 13.3). Cancer registry quality indicators were excellent.

Key words: Cancer Registry. Head and neck cancer incidence.

\section{Introducción}

El análisis de la incidencia es una herramienta fundamental para estimar la magnitud y distribución de un problema de salud. Desde el punto de vista de la organización sanitaria, proporciona los datos necesarios para evaluar las necesidades asistenciales, planificar el uso de recursos y plantear estrategias preventivas prioritarias, como las campañas de diagnóstico precoz. Desde el punto de vista científico, el análisis de la incidencia es a su vez básico para generar hipótesis etiológicas, objetivo señalado ya desde sus primeras ediciones en las publicaciones de referencia sobre incidencia del cáncer' ${ }^{1}$.

El cáncer es la segunda causa de mortalidad en los países desarrollados y es un problema de salud de trascendencia incuestionable. El cáncer de cabeza y cuello (CCC) representa el $5-10 \%$ de todos los procesos malignos ${ }^{2}$. En España origina aproximadamente el $5 \%$ de las muertes por cáncer ${ }^{3}$. A pesar de todo, las publicaciones sobre estudios de incidencia del CCC en España son escasas.

El Registro de Tumores o Registro de Cáncer es la fuente de datos básica para el análisis de la incidencia de esta enfermedad. Existen Registros de Tumores en el ámbito hospitalario (Registro de Tumores Hospitalario, RTH) y Registros de Cáncer de base poblacional (Registro de Cáncer Poblacional, RCP). Su objetivo es recoger de forma exhaustiva y continuada los datos básicos sobre todos los casos de cáncer. En los RTH se recogen todos los casos diagnosticados o tratados en el hospital, cualquiera que sea su procedencia. Las fuentes de datos de los RCP son tanto hospitalarias (servicios de anatomía patológica, oncología, hemato- logía, otros servicios clínicos, archivo de historias clínicas y servicio de admisión), como extrahospitalarias (registros de mortalidad, atención primaria y otros registros de tumores). Se mantienen también, Registros de Tumores o bases de datos de pacientes oncológicos, en servicios clínicos implicados en el manejo de esta patología. Por último existen entidades supranacionales como la Asociación Internacional de Registros de Cáncer (IACR, International Association of Cancer Registries), o en un ámbito más próximo la Red Europea de Registros de Cáncer (ENCR, European Network of Cancer Registries).

El objetivo del presente estudio es evaluar la incidencia del CCC en un área de salud mediante la explotación de un Registro de CCC, a la vez que analizar la calidad del mismo registro.

\section{Material y métodos}

El hospital Santa Bárbara (www.hsbarbara.com) es un centro de nivel 1 que cubre el Área de Salud de Puertollano, en la provincia de Ciudad Real, ubicada en el límite sur del territorio del actual Servicio de Salud de Castilla-La Mancha (SESCAM). Al igual que otras zonas de Castilla-La Mancha, es un área geográficamente extensa, con algunas poblaciones situadas a más de $100 \mathrm{~km}$ de distancia del centro hospitalario. El hospital Santa Bárbara es el centro sanitario público de referencia de esta población, no existiendo centros privados en el área que cubran estas funciones.

En octubre de 1999 se creó en el servicio de ORL un registro de CCC con el fin de facilitar el seguimiento 
de estos pacientes, así como la realización de estudios observacionales. Dicha iniciativa se debió además a la no existencia de registros formales de este tipo en nuestro medio próximo, así como a la ausencia de otros elementos que propiciaran un manejo mejor documentado de estos pacientes (informatización de las historias clínicas, protocolos de canalización, comité de tumores, servicios de oncología...). Las fuentes de datos para la identificación de los casos han sido las propias, clínicas, del servicio de ORL, la base de datos del servicio de anatomía patológica y otras referencias administrativas como los documentos de canalización para tratamientos externos que necesariamente son autorizados por el servicio de ORL. El registro no incluye pacientes no residentes en el área de salud.

Se diseñó un estudio longitudinal y prospectivo para determinar la incidencia del CCC en la población atendida en un período de dos años (enero 2000- diciembre 2001). La recogida de datos se realizó en el marco del Registro de CCC. Para el presente estudio se decidió utilizar solamente las variables básicas: sexo, edad, localización y estadiaje e histopatología.

Para las localizaciones en cabeza y cuello se ha seguido la clasificación de la "Union Internationale Contre le Cancer" (UICC), 19974, excepto por la inclusión de los linfomas que debutaron en cabeza y cuello. Se tomó esta decisión por razones prácticas de manejo diagnóstico ya que su forma de presentación es como masa cervical u otros síntomas ORL según la localización. Al expresar los resultados se especifica en cada caso la inclusión o no de los linfomas. Se han excluido los cánceres cutáneos no melanoma. El estadiaje TNM expresado es clínico (exploración física y estudio de imagen, habitualmente TC helicoidal con contraste).

Los datos demográficos se obtuvieron de la página web del Instituto Nacional de Estadística (www.ine.es) a través de su herramienta de consulta "inebase". Se consultaron también los Registros de Mortalidad y se solicitó información poblacional a la gerencia de atención primaria, obtenida a través de la Tarjeta Sanitaria. El área de salud tiene una población más o menos estable alrededor de 80.000 habitantes (población al inicio del estudio a 1 enero de 2000: 80.962; población media del período de estudio: 79.902). Se trata de una población de edad más avanzada que la población estándar mundial (Fig. 1) con una mediana de edad de 37 años (frente a 29 años de la estandar). Algo menos del $20 \%$ de la población del área es mayor de 65 años.

Se han tomado como referencias la normativa de la Agencia Internacional para la Investigación sobre el Cáncer (IARC, International Agency for the Research on Cancerl $)^{5}$ para los Registros de Tumores y la codifi-

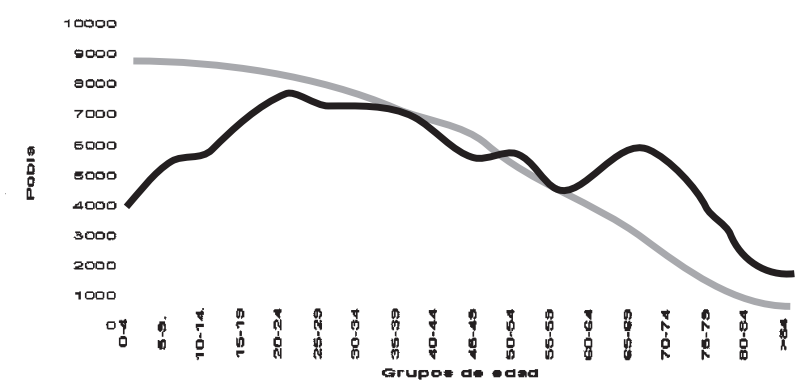

Fig. 1. Gráficos poblacionales. En abcisas se muestran los grupos quinquenales de edad y en ordenadas el número de habitantes. Los gráficos de líneas muestran la distribución por edades de la población del área de Puertollano estandarizada (línea oscura), y la población estándar mundial (línea clara).

cación según la Clasificación Internacional de Enfermedades para Oncología (CIE-O) de la $\mathrm{OMS}^{6}$. Dicha estandarización es básica para posibilitar la agrupación de datos y los estudios comparativos.

Para la evaluación de los criterios de calidad de registro es suficiente la explotación de las mismas variables señaladas arriba. Estos criterios son: el porcentaje de casos conocidos o identificados por certificados de defunción o registros de mortalidad, el porcentaje confirmado histológicamente, el porcentaje de pacientes de edad no conocida y el porcentaje de casos etiquetados como Tumor de Origen Desconocido.

\section{Resultados}

En el período de estudio se presentaron en total 48 tumores malignos de cabeza y cuello, lo cual corresponde a una incidencia acumulada o "riesgo" de $0,06 \%$ en dos años $(0,05 \%$ excluyendo 7 linfomas). La tasa bruta de incidencia anual fue de 30 tumores por 100.000 habitantes o de 25,7 excluyendo los linfomas.

Por localizaciones, 11 casos fueron de cavidad oral y orofaringe, 21 de laringe e hipofaringe, 7 fueron cánceres de tiroides, los mencionados 7 linfomas con debut en cabeza y cuello y 2 tumores malignos de glándulas salivares. La sublocalización más frecuente fue la supraglotis con 9 casos $(18,8 \%)$, seguida de la glándula tiroides con $7(14,6 \%)$ y la glotis con 6 $(12,5 \%)$. Hubo 5 tumores de lengua libre $(10,4 \%)$. De los linfomas, 5 debutaron en el cuello, uno en región parotídea y el último en fosa nasal (Tabla I).

El tipo histológico más frecuente fue el carcinoma epidermoide con 32 casos (66,7\%). Los tumores de la vía aerodigestiva superior fueron todos carcinomas 
TABLA I

\begin{tabular}{|c|c|c|c|c|}
\hline \multicolumn{5}{|c|}{ Localizaciones y sublocalizaciones de los casos } \\
\hline Localización & Sublocalización & Frecuencia & \multicolumn{2}{|c|}{ Porcentaje } \\
\hline \multirow{2}{*}{ Fosas nasales y senos paranasales } & & & & 2,1 \\
\hline & Fosa nasal & 1 & 2,1 & 209 \\
\hline \multirow{4}{*}{ Cavidad oral } & Lengua & 5 & 10,4 & \\
\hline & Suelo de boca & 2 & 4,2 & \\
\hline & Paladar duro & 1 & 2,1 & \\
\hline & Trígono retromolar & 2 & 4,2 & \\
\hline \multirow[t]{3}{*}{ Faringe } & & & & 6,3 \\
\hline & Base de lengua & 1 & 2,1 & \\
\hline & Hipofaringe & 2 & 4,2 & \\
\hline \multirow[t]{5}{*}{ Laringe } & & & & 39,5 \\
\hline & Supraglotis & 9 & 18,7 & \\
\hline & Glotis & 6 & 12,5 & \\
\hline & Subglotis & 1 & 2,1 & \\
\hline & (Transglóticos) & 3 & 6,2 & \\
\hline \multirow[t]{2}{*}{ Glándulas salivares } & & & & 6,2 \\
\hline \multirow{3}{*}{\multicolumn{2}{|c|}{$\begin{array}{l}\text { Cáncer de glándula tiroides } \\
\text { Cuello (linfomas de presentación cervical) }\end{array}$}} & 3 & 6,2 & \\
\hline & & 7 & 14,6 & 14,6 \\
\hline & & 5 & 10,4 & 10,4 \\
\hline \multicolumn{2}{|l|}{ Total } & 48 & 100,0 & 100,0 \\
\hline
\end{tabular}

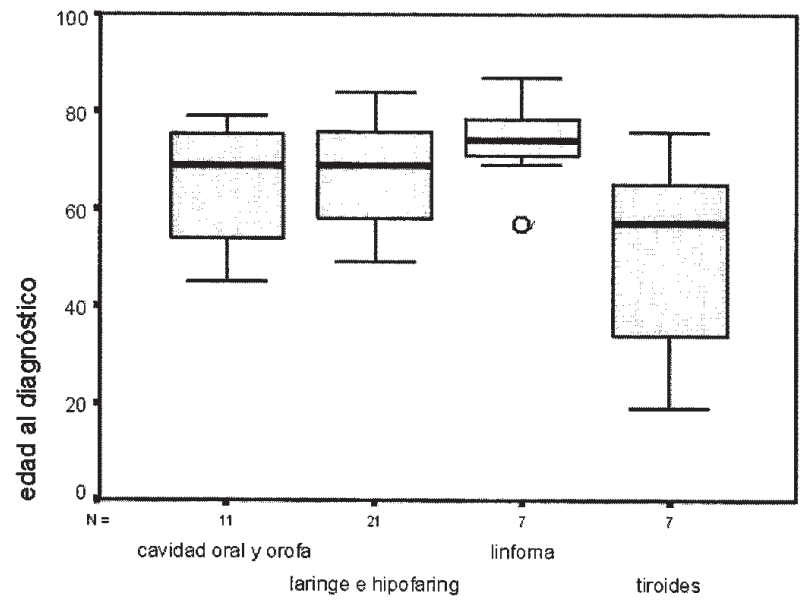

Region

Fig. 2. Gráficos de caja con la distribución de edades por localizaciones. Para los tumores de la vía aerodigestiva superior encontramos el rango esperable centrado alrededor de la sexta y séptima décadas de la vida, unas edades algo mayores para los pacientes con linfomas, excepto por un paciente más joven (un varón de 57 años, que fue precisamente una enfermedad de Hodgkin); encontramos para los cánceres de tiroides, una población en general más joven (media 50 años), pero con una dispersión máxima (DE 21,8 años) y un rango de edades más amplio (entre un mínimo de 19 años y un máximo de 76 años). epidermoides, excepto un caso de adenocarcinoma de paladar duro y el mencionado linfoma de fosa nasal. El linfoma no Hodgkin fue el segundo tipo histológico más frecuente con 6 casos (12,5\%), seguido del carcinoma papilar de tiroides con 4 casos $(8,3 \%)$.

El rango de edad del grupo global fue de 68 años, siendo el paciente más joven de 19 años (un varón con un carcinoma papilar de tiroides) y el mayor de 87 años (una mujer con un linfoma no Hodgkin). La media de edad fue 65,6 años, con una desviación estándar de 14,6 años. El 62,5\% de los pacientes oncológicos fueron mayores de 64 años, y sólo el $4 \%$ menores de 35 años. El grupo quinquenal de edad de máxima incidencia fue el de los 75 a 79 años, con 12 casos (25\%). Se muestra en la Figura 2 una comparación de distintas localizaciones respecto a la edad.

Respecto a la distribución por sexos (Fig. 3), globalmente la razón fue de tres a uno a favor de los varones. Encontramos un claro predominio del sexo masculino para el cáncer de laringe $(20 / 1)$ y cocientes más ajustados para otras localizaciones (2,7 en cavidad oral-orofaringe y 1,3 en los linfomas). La razón de sexos sólo se invierte para el cáncer de tiroides, que es más frecuente en mujeres (razón varones/mujeres de 0,75).

El cáncer de laringe fue el tumor más frecuente, con un total de 19 casos (tasa bruta de incidencia de 11,8 casos por 100.000 habitantes y año). De ellos, 9 fue- 


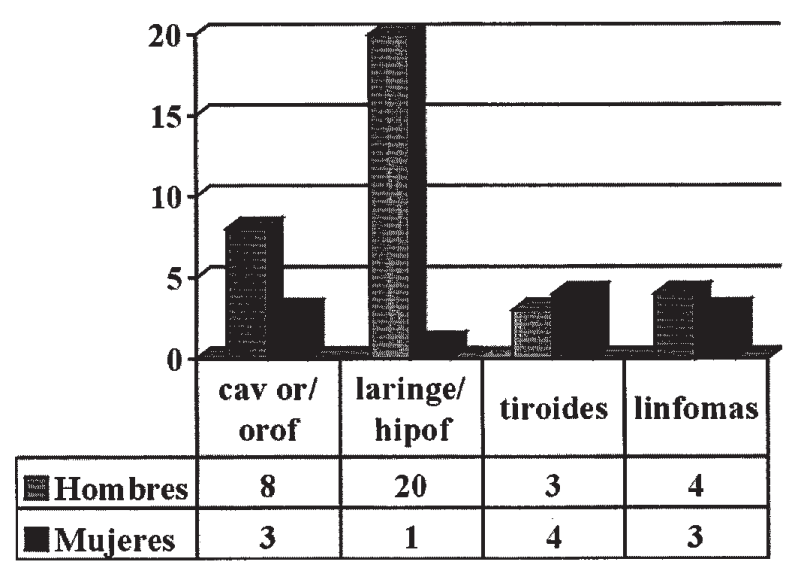

Fig. 3. Distribución por localizaciones y sexo. En la tabla se muestra el número de casos.

ron supraglóticos y 10 gloto-subglóticos (6 glóticos, 3 transglóticos y 1 subglótico). Entre los tumores supraglóticos, el $55 \%$ se presentaron con adenopatías al diagnóstico, pero el $100 \%$ de los casos fueron clasificados como estadios avanzados (III-IV). Los tumores gloto-subglóticos fueron más frecuentemente clasificados como $\mathrm{Tl}$ (en el $40 \%$ de los casos); el $90 \%$ de ellos se presentaron sin adenopatías clínicas, pero el 50\% fueron estadios avanzados, fundamentalmente por la T, debido a fijación de la cuerda vocal (T3). Los 2 tumores de hipofaringe fueron un TIN3 y un T4N0, ambos de seno piriforme.

Respecto a las lesiones de cavidad oral y orofaringe, la distribución fue heterogénea. La sublocalización más frecuente fue la lengua libre con 4 casos (TINO, T2N0, T2N2, T4N3). Los demás fueron 2 lesiones de suelo de boca, ambas localmente avanzadas (T4N1, T4N3), 2 de trígono retromolar (T3N2, T4NO), un adenocarcinoma de paladar duro (T1NO) y un carcinoma epidermoide de base de lengua (T2N3).

El cáncer de la glándula tiroides tuvo una tasa bruta de incidencia anual de 4,4 casos por 100.000 habitantes. Histopatológicamente, encontramos 4 cánceres papilares, 2 foliculares y un carcinoma medular de tiroides.

Todos los tumores de cabeza y cuello fueron $\mathrm{MO}$ al diagnóstico.

\section{Discusión}

El análisis de la población es un paso previo necesario para el estudio de la incidencia de las enfermedades. En nuestra área de salud, llama la atención un bache poblacional entre los 55 y 65 años, cuyo análisis sería complejo, pero que responde sin duda a peculiaridades sociológicas e históricas del área. Precisamente en dicho rango de edades se encuentra el pico de incidencia esperable para los tumores de cabeza y cuello. Los sesgos que esta distribución por edades podría crear, se salvan mediante la estandarización de tasas. En relación con la incidencia, la tasa bruta es el dato más interesante respecto a la planificación sanitaria. Esta tasa puede ser difícil de estimar si no existe un RCP y, en cualquier caso, la estimación es compleja si el sistema no es relativamente cerrado, circunstancia a la que se aproxima nuestra área de salud. Aunque no podemos confirmar de forma directa la exhaustividad del registro, y de hecho existe un evidente sesgo de selección de los pacientes, encontramos indicios para sospechar que los casos incluidos se aproximan a la totalidad de los producidos entre los residentes en el área. Se han comentado arriba las razones geográficas y de comunicaciones, la organización de la asistencia sanitaria, así como por el esfuerzo organizativo realizado. Esto es particularmente cierto para los tumores más característicamente otorrinolaringológicos, como el cáncer de laringe, para el cual, como se analiza más abajo, la incidencia registrada se encuentra en el rango de incidencia de nuestro medio. Podemos en cualquier caso considerarlos como datos mínimos de incidencia. Por todo ello hemos considerado que existen unas condiciones adecuadas para realizar estudios de incidencia. Así pues, aunque se trata de un RTH, posiblemente es asimilable a un RCP específico de cabeza y cuello en el área de salud de Puertollano. Por esta razón nos hemos permitido la presentación de tasas brutas y la estandarización de tasas respecto a la población residente y no respecto a la población atendida, dato que no podemos conocer.

El cáncer de laringe es el tumor maligno más frecuente en cabeza y cuello. Es la segunda neoplasia maligna más frecuente en las vías respiratorias después del cáncer de pulmón 7 . Nuestra casuística refleja esta prevalencia, siendo el tumor que más frecuentemente encontramos. El cáncer de laringe en mujeres sigue siendo muy raro en nuestra área ${ }^{8}$. El $75 \%$ de los tumores de la vía aerodigestiva superior se presentaron en estadios avanzados.

Notablemente, para el cáncer de tiroides y a pesar del reducido número de casos, encontramos, posiblemente fruto del azar, una distribución que refleja la incidencia de los distintos tipos histopatológicos?, excluyendo el carcinoma indiferenciado o anaplásico. Dicha incidencia, con grandes diferencias geográficas, es de $40-80 \%$ de carcinomas papilares, $15-40 \%$ de foliculares, y $5 \%$ de carcinomas medulares de tiroides. 


\section{J. Granell Navarro y A. Puig Rullán}

Nuestros 7 casos se distribuyeron en una relación 4:2:1. La razón de sexos, sin embargo, suele ser más favorable a las mujeres (2-4:1) de la que hemos encontrado (1,3:1). La edad media de diagnóstico es de 45 años para los papilares $(40,8$ años en nuestros 4 pacientes), 55 años para los foliculares (65 en nuestros datos) y 50-60 para el carcinoma medular esporádico (no familiar); nuestro paciente tenía 57 años.

La discusión sobre la incidencia de los linfomas no es procedente, ya que hemos considerado sólo los que debutaron en cabeza y cuello. El interés de su inclusión en el protocolo diagnóstico de los procesos malignos de cabeza y cuello es el de garantizar un manejo diagnóstico adecuado, particularmente en lo que se refiere a la biopsia prematura de las masas cervicales. Es destacable que considerados en conjunto, los linfomas representan un importante porcentaje de las lesiones malignas que se presentan en cabeza y cuello.

Hemos considerado interesante la comparación de tasas con otras poblaciones. Para ello hemos realizado un ajuste de las mismas respecto a la población estándar mundial. Las tasas ajustadas en los diversos países las hemos obtenido de la base de datos GLOBOCAN 2000 de la IARC ${ }^{10}$. Se trata de estimaciones para el año 2000 basadas en la población estimada a mediados de ese año y los datos más recientes disponibles sobre incidencia, generalmente de 3 o 4 años antes.

Para el cáncer de laringe, la tasa ajustada de incidencia es de 5,33 casos/año por 100.000 habitantes en Estados Unidos para varones. En Europa, las tasas más bajas son las de los países nórdicos (2,09 en Suecia, 3,22 en Noruega). Los valores más altos, por el contrario, los encontramos en los países mediterráneos $(10,82$ en Italia y 10,17 en Francia), siendo la tasa española de las más elevadas: 14,07. En nuestra área de salud, la tasa ajustada de incidencia específica para varones es de 13,3 casos por 100.000 habitantes $y$ año. Alcanza por tanto valores superiores a las tasas norteamericana y nórdicas y similar a las de nuestro entorno: 14,2 en la Comunidad Valenciana ${ }^{11}$ y 14,5 en Navarra' ${ }^{12}$.

Los datos de incidencia de GLOBOCAN para España son estimaciones. Utilizan modelos de estimación basados en los datos disponibles de mortalidad e incidencia de los Registros de Cáncer ante la ausencia de datos de incidencia a nivel nacional. Es evidente que esto señala carencias en los Registros de Cáncer de nuestro país. El uso de los Registros de Mortalidad para estimaciones de incidencia merece un comentario aparte por los problemas que genera. En primer lugar, la razón mortalidad/incidencia debe ser considerada con cautela, ya que se ve influida tanto por la supervivencia de los pacientes como por la calidad de los cer- tificados de defunción. Esto es así a pesar de que en el caso del CCC el resultado de un tratamiento inefectivo suele conllevar al fallecimiento por la enfermedad no controlada (es poco habitual el éxitus por otras causas), lo cual aparentemente proporciona una variable resultado fácil de medir (fallecimiento por cáncer) y por tanto, en principio, una causa fácil de certificar. Hemos consultado la mortalidad específica por cáncer en nuestra área y hemos encontrado los registros manifiestamente escasos respecto a los datos que manejamos. Por otro lado, una forma de estimar la exahustividad de los Registros de Cáncer se basa precisamente en el porcentaje de casos identificados por el certificado de defunción.

Otros parámetros que se manejan como indicadores de calidad de los Registros de Tumores son el porcentaje de casos confirmados histológicamente y el porcentaje de casos de edad desconocida. Si consideramos estos indicadores en nuestro registro, encontramos que todos nuestros casos fueron confirmados histológicamente; en ningún caso se desconocía la edad del paciente y en ningún caso se tuvo conocimiento del mismo recurriendo al certificado de defunción. Tampoco ningún caso fue registrado como Tumor de Origen Desconocido. Por tanto, los indicadores habituales fueron excelentes, como es esperable de un registro prospectivo de un servicio clínico. En este sentido, es lógico que este tipo de registro sea en general más completo, aunque menos exhaustivo, que los Registros de Tumores estándar, ya que son mantenidos por personal facultativo directamente implicado en el manejo de estos pacientes. Los índices de fiabilidad mejoran por tanto los de los RCP reconocidos por el Ministerio de Sanidad y Consumo. Por las razones señaladas parece deseable disponer de registros de incidencia en el nivel asistencial para mejorar la calidad.

La epidemiología es una rama de la medicina preventiva y salud pública encargada del estudio de la distribución y la dinámica de las enfermedades en las poblaciones humanas. No es por tanto una competencia directa del otorrinolaringólogo el control de la incidencia de las enfermedades. Sin embargo, consideramos que los servicios de ORL se encuentran en una excelente situación para mantener bases de datos formales y sistemáticas de los pacientes oncológicos de cabeza y cuello en sus áreas de influencia.

Correspondencia:

Dr. J. Granell Navarro

Paseo de la Reina Cristina, 16 - $4^{\circ}$

E-28014 Madrid

igranelln@seorl.net 


\section{Bibliografía}

1. Doll R, Payne P, Waterhouse J. Cancer Incidence in Five Continents Vol I. UICC. Springer-Verlag, Berlin-Heidelberg-New York, 1966

2. Davis WE, Zitsch RP. Statistics of head and neck cancer. En: Thawley SE, Panje WR, Batsakis JG, Lindberg RD: Comprehensive management of head and neck tumors. WB Saunders Company, 2nd ed. Philadelphia, 1999; 283-95

3. Mortalidad por cáncer en España, 1998. Area de epidemiología ambiental y cáncer. Centro Nacional de Epidemiología. Disponible en: http://193.146.50.130/cancer/mort98.txt

4. UICC. TNM Classification of malignant tumors. 5th ed. Wiley-Liss. New York, 1997

5. Jensen OM, Parkin DM, MacLennan R, Muir CS, Skeet RG. Cancer Registration: Principles and Methods. IARC Scientific Publications no 95. Lyon, 1991

6. Percy C, Van Holten V, Muir C. Clasificación Internacional de Enfermedades para Oncología, $2^{\mathrm{a}}$ edición. Ginebra. Organización Mundial de la Salud, 1995

7. Cattaruzza MS, Maisonneuve P, Boyle P. Epidemiology of laryngeal cancer. Eur J Cancer B Oral Oncol 1996; 32B(5):293-305

8. Gallus S, Bosetti C, Franceschi S, Levi F, Negri E, La Vecchia C. Laryngeal cancer in women: tobacco, alcohol, nutritional, and hormonal factors. Cancer Epidemiol Biomarkers Prev. 2003 Jun; 12(6):514-7

9. Grebe SKG, Hay HD. Clinical evaluation of thyroid tumors. En: Thawley SE, Panje WR, Batsakis JG, Lindberg RD: Comprehensive management of head and neck tumors. WB Saunders Company, 2nd ed. Philadelphia, 1999; 1964-1709

10. Ferlay J, Bray F, Pisani P, Parkin DM. GLOBOCAN 2000: Cancer incidence, mortality and prevalence worldwide, version IARC CancerBase No.5. IARC Press. Lyon, 2001. Disponible en: www-dep.iarc.fr/globocan/globocan.html

11. Servicio de epidemiología. Consellería de Sanitat de la Comunidad Valenciana. Disponible en: http:// dgsp. san. gra.es/sccc/epidemiologia/tumores/tumores.htm

12. Registro de Cáncer de Navarra. Incidencia del Cáncer en Navarra. 1988-92. An Sist Navar 1998; 21(1):65-75. 